\title{
ON AN APPLICATION OF GUTH-KATZ THEOREM
}

\author{
Alex Iosevich, Oliver Roche-Newton and Misha Rudnev
}

\begin{abstract}
We prove that for some universal $c$, a non-collinear set of $N>\frac{1}{c}$ points in the Euclidean plane determines at least $c \frac{N}{\log N}$ distinct areas of triangles with one vertex at the origin, as well as at least $c \frac{N}{\log N}$ distinct dot products.

This in particular implies a sum-product bound
\end{abstract}

for a discrete $A \subset \mathbb{R}$.

$$
|A \cdot A \pm A \cdot A| \geq c \frac{|A|^{2}}{\log |A|}
$$

\section{Introduction}

In 1946 P. Erdős [6] posed what later became known as the Erdös distance problem. The question was to prove that a plane set of $N$ distinct points determines at least $N^{1-o(1)}$ distinct distances. Some 65 years later, after a large number of partial results and insightful ideas, the problem was solved by Katz and Guth [11], who proved that $N>\frac{1}{c}$ points in the plane determine more than $c \frac{N}{\log (N)}$ distinct distances. See, for example, $[1,9]$ and the references contained therein for the previous work on this influential conjecture and connections with other problems in geometric combinatorics.

The Guth-Katz proof is based to a significant extent on the polynomial method which was introduced by Dvir [3] in the context of the Kakeya problem over finite fields, and developed extensively by Guth and Katz [10], Guth [8], Elekes et al. [5] and others. The key point of the Guth-Katz proof of the Erdös distance conjecture is the following result based on the aforementioned polynomial method.

Theorem 1.1. A set of $N^{2}$ straight lines in $\mathbb{R}^{3}$, such that

(i) no more than $O(N)$ lines are concurrent,

(ii) no more than $O(N)$ lines lie in a single plane,

(iii) no more than $O(N)$ lines lie in a single doubly ruled surface, have $O\left(N^{3} \log N\right)$ pair-wise intersections.

Remark 1.1. It is well known that there are only two doubly ruled non-plane surfaces in $\mathbb{R}^{3}$ : hyperbolic paraboloid, and single-sheeted hyperboloid, both having degree two (see, e.g., [14]).

In this paper, we shall see that Theorem 1.1 implies the following results.

Theorem 1.2. There exists a universal $c>0$ such that a set of $N>\frac{1}{c}$ non-collinear points in $\mathbb{R}^{2}$ determines at least $c \frac{N}{\log N}$ distinct areas of triangles with one vertex at the origin.

Received by the editors March 13, 2011.

2000 Mathematics Subject Classification. 68R05,11B75. 
We also have the following sum-product type inequality.

Corollary 1.1. Let $A$ be a finite subset of reals. Then

$$
|A \cdot A \pm A \cdot A| \gg \frac{|A|^{2}}{\log |A|} .
$$

Above and throughout, $|A|$ denotes cardinality of a discrete set $A$; the notation $X \ll Y$ means that there exists $c>0$, such that $X \leq c Y$. Besides, $X \gg Y$ means $Y \ll X$, and $X \approx Y$ means $X \ll Y$ and $Y \ll X$.

Counting areas of triangles with one vertex at the origin can be easily converted to counting dot products. It is an immediate consequence of the forthcoming proof of Theorem 1.2 that its statement can be generalized as follows.

Theorem 1.3. Let $P, P^{\prime} \subset \mathbb{R}^{2}$ such that $|P|=\left|P^{\prime}\right|=N$ and that both $P$ and $P^{\prime}$ are not contained in a single line. Let $\mathcal{A}\left(P, P^{\prime}\right)$ denote the set of areas of triangles with one endpoint at the origin, one at a point in $P$, and the other at a point in $P^{\prime}$. Then

$$
\left|\mathcal{A}\left(P, P^{\prime}\right)\right| \gg \frac{N}{\log (N)} .
$$

Theorem 1.3 has an immediate corollary.

Corollary 1.2. Let $P \subset \mathbb{R}^{2}$ with $|P|=N$. Let

$$
\Pi(P)=\{v \cdot w: v, w \in P\} .
$$

be the set of dot products generated by pairs of vectors from $P$. Then

$$
|\Pi(P)| \gg \frac{N}{\log (N)} .
$$

Observe that our results (as well as the Guth-Katz solution of the Erdös conjecture) are optimal up to logarithmic factors, while Theorem 1.1 is optimal up to constants.

The problems discussed in this paper have been studied quite extensively in recent years. Pinchasi [13] proved that for a set $P$ of $N$ non-collinear points there exist two points $A, B \in P$ such that the areas of triangles $A B C$, with $C \in P$ have at least $\left\lfloor\frac{N-1}{2}\right\rfloor$ distinct values. However, as far as the triangles with one vertex at the origin are concerned we are not aware of a result that would claim to guarantee more than $c N^{\frac{2}{3}}$ distinct areas, the latter estimate being a direct application of the Szemerédi-Trotter theorem [15]. One may contrast this with the Erdös distance problem which had had a series of incremental improvements over the past years, due to more elaborate applications of the Szemerédi-Trotter theorem and arithmetic combinatorics methods. See [11] and [1] and the references contained therein.

As for the inequality (1), the estimate with $|A|^{\frac{3}{2}}$ in the right-hand side follows once again from the Szemerédi-Trotter theorem, (see, e.g., [16]), but we are not aware of better bounds.

In the continuous setting, both problems are studied in [7]. For finite field versions of these problems see, for example, [12] and [2]. In all of these instances, the exponents are not optimal. 


\section{Proof of the main results}

Proof of Theorems 1.2 and 1.3. Let $P \subset \mathbb{R}^{2}$, be a discrete non-collinear point set, with the cardinality $|P|=N$. We can clearly assume that there is no line $l$ containing more than $\frac{N}{2}$ points of $P$ (recall that the whole of $P$ cannot be supported on a line), or there are at least $\left\lfloor\frac{N}{2}\right\rfloor-1$ triangles with different areas.

Before we begin, let us rotate the point set $P$ around the origin to ensure that no points of $P$ lie on the coordinate axes and each vertical line $x=c$ supports no more than one point of $P$. This is done only in order to be able to specify a generic projection' of $\mathbb{R}^{4}$ onto $\mathbb{R}^{3}$ in the ensuing argument as the one along the $x_{4}$-axis.

We now follow the energy approach, looking at the number of quadruples that solve

$$
v^{1} \wedge v^{2}=v^{3} \wedge v^{4}
$$

where $v^{i}, i=1, \ldots, 4$ are elements of $P$, and $\wedge$ denotes the standard oriented vector product. Let us restrict the equation (2) to the case of non-collinear $\left(v^{1}, v^{2}\right)$ and noncollinear $\left(v^{1}, v^{3}\right)$, as well as the case when one rotates from $v^{1}$ to $v^{2}$ in the positive direction.

Under this introductory set of assumptions, let $n(s)$ be the number of occurrences of the value $s>0$ of the wedge product. Then we have

$$
\sum_{s>0} n(s) \gg N^{2}
$$

We are going to show that

$$
E=\sum_{s>0} n^{2}(s) \ll N^{3} \log N
$$

and Theorem 1.2 will follow by the Cauchy-Schwarz inequality.

Remark 2.1. As is noted by Guth and Katz in [11], the idea of using energy followed by the argument of rotations was initially introduced by Elekes and Sharir in [4].

We shall demonstrate (3) by deducing it from Theorem 1.1. The remainder of this paper is dedicated to this reduction.

Equation (2) has a solution if and only if there is a linear area-preserving transformation $T$, such that $T\left(v^{1}\right)=v^{3}$ and $T\left(v^{2}\right)=v^{4}$. One direction is obvious, and the only if direction follows from the fact that for any two non-collinear pairs of vectors there is a linear transformation mapping one pair to the other, and if the areas of the corresponding triangles are equal, then the transformation is area-preserving.

Consider families $\mathcal{T}_{i j}$ of linear area-preserving transformations $T_{i j}=T_{v^{i}, v^{j}}$, acting so that $T_{i j}\left(v^{i}\right)=v^{j}$, for positively oriented pairs of non-collinear vectors $v^{i}, v^{j} \in P$. Then, given a basis of $\mathbb{R}^{2}, T_{i j}$ is represented by a matrix $A_{i j}$ in $S L_{2}(\mathbb{R})$. Since $v^{i}, v^{j}$ are non-collinear, they themselves can be considered as a basis, in which case, since $T[(1,0)]=(0,1)$,

$$
A_{i j}=A_{i j}(t)=\left(\begin{array}{cc}
0 & -1 \\
1 & t
\end{array}\right), \quad \text { for } t \in \mathbb{R}
$$


In the standard basis, $T_{i j}$ is represented by the matrix $\tilde{A}_{i j}=C_{i j} A_{i j} C_{i j}^{-1}$, where $C_{i j}$ is the transition matrix: given $v^{i}=(a, b), v^{j}=(c, d)$ we have

$$
\begin{aligned}
\tilde{A}_{i j} & =\frac{1}{a d-b c}\left(\begin{array}{ll}
a & c \\
b & d
\end{array}\right)\left(\begin{array}{cc}
0 & -1 \\
1 & t
\end{array}\right)\left(\begin{array}{cc}
d & -c \\
-b & a
\end{array}\right) \\
& =\frac{1}{a d-b c}\left(\begin{array}{ll}
c d+a b-b c t & -c^{2}-a^{2}+a c t \\
d^{2}+b^{2}-b d t & -c d-a b+a d t
\end{array}\right) .
\end{aligned}
$$

Therefore, in the standard basis, the family of transformations $\mathcal{T}_{i j}$ corresponds to a straight line ${ }^{1}$ inside a quadric hypersurface $H \subset \mathbb{R}^{4}$. The equation for $H$ is $x_{1} x_{4}-x_{2} x_{3}=1$.

Remark 2.2. This is the only fact about the group $S L_{2}(\mathbb{R})$ which is used in this argument. Guth and Katz dealt with the Euclidean group $S E_{2}(\mathbb{R})$. Both are threedimensional Lie groups, and in both cases the problem in question enables a simple incidence parameterisation ${ }^{2}$. Note that the fact that the two groups have the same dimension is no longer true in dimension $d>2$.

A solution of Equation (2) corresponds to the intersection of a pair of such straight lines. Let $L$ denote the family of these $\approx N^{2}$ lines.

We are now going to implement a generic projection argument in $\mathbb{R}^{4}$ by projecting $H$ to $\mathbb{R}^{3}$ and showing that the conditions of Theorem 1.1 in $\mathbb{R}^{3}$ are satisfied. In order to do this rigorously, let us make a few observations about the lines in $L$.

First, no more than $N$ lines from $L$ can be concurrent. Indeed, otherwise there would be a $v^{i} \in P$, which by the same linear transformation $T$ would be mapped into more than one point $v^{j} \in P$.

Second, observe that after the original random rotation none of $a, b, c, d$ equals zero, and therefore, by (5), no line of $L$ is contained in the section $x_{1}=0$ of $H$.

This enables us just to consider the projection of $H$ on the $\left(x_{1}, x_{2}, x_{3}\right)$-space, in the standard basis. This projection is one-to one, as long as we restrict $x_{1}>0$, and the latter restriction does not lead to a to loss of generality, since by (5) there are no lines in the $x_{4}$-direction, nor are there lines contained inside the section $x_{1}=0$ of $H$.

The lines (5) project to the $\left(x_{1}, x_{2}, x_{3}\right)$ into a family $L^{\prime}$ of $\approx N^{2}$ lines

$$
\left(x_{1}, x_{2}, x_{3}\right)(t)=\frac{1}{a d-b c}\left[\left(-a c-b d,-c^{2}-d^{2}, a^{2}+b^{2}\right)+t(-b c,-c d, a b)\right] .
$$

Let us verify the non-degeneracy conditions $(i i),(i i i)$. Let us start with $(i i i)$, where we essentially copy the corresponding part of the proof of Proposition 2.3 in [11].

Let us fix $(a, b)$ and vary $(c, d)$ continuously, under the constraints $a, b, c, d, a d-b c \neq$ 0 . Let us call $L_{a, b}$ the continuous family of these lines. Let $S^{\prime}$ be some non-plane doubly ruled surface. If more than $O(1)$ lines of a single ruling of $S^{\prime}$ lies in some $L_{a, b}$, then all the lines from this ruling must lie in $L_{a, b}$. Besides, for $(a, b) \neq\left(a^{\prime}, b^{\prime}\right)$, the sets $L_{a, b}$ and $L_{a^{\prime}, b^{\prime}}$ are disjoint (this is the case for the original lines in $H$ and the

\footnotetext{
${ }^{1}$ Geometrically it is easy to see. Let $v^{i}=(1,0), v^{j}=(0,1)$, the standard basis unit vectors. A transformation $T_{i j}$ would rotate $(1,0)$ into $(0,1)$, while the image of $(0,1)$ would be anywhere on the vertical ray beginning at $(-1,0)$.

${ }^{2}$ For some discussion why this is the case see http://terrytao.wordpress.com/2011/03/05/lines-inthe-euclidean-group-se2/\#more-4676.
} 
projection of $L$ onto $L^{\prime}$ is one-to-one). Thus if more than $O(1)$ lines from a ruling of $S^{\prime}$ lie in some $L_{a, b}$, no lines of this ruling lie in any other $L_{a^{\prime}, b^{\prime}}$.

Indeed, consider the space of lines in $\mathbb{R}^{3}$. By (6), since $a b \neq 0$, we can only look at the lines that are not contained in planes $x_{3}=$ const. These lines are defined by their initial point $\left(x_{1}, x_{2}, 0\right) \cong \mathbb{R}^{2}$ and the direction vector $\left(d_{1}, d_{2}, 0\right) \cong \mathbb{R}^{2}$. Thus given $(a, b)$, equations (6) can be rewritten as $P_{1}\left(x_{1}, x_{2}, d_{1}, d_{2}\right)=P_{2}\left(x_{1}, x_{2}, d_{1}, d_{2}\right)=0$ for some $O(1)$ degree polynomials $P_{1}$ and $P_{2}$. On the other hand, a single ruling of $S^{\prime}$ corresponds to a straight line in $\mathbb{R}^{4}$. If this line intersects the zero set of $P_{1}$ and $P_{2}$ more than $O(1)$ times, it is contained in it.

Hence, given $S^{\prime}$, since there are only two families of lines, foliating $S^{\prime}$, there may be up to two exceptional points $v^{i}=(a, b) \in P$, such that $S^{\prime}$ contains up to $N$ lines representing the families of transformations $\mathcal{T}_{v^{i}, v^{j}}=\mathcal{T}_{(a, b),(c, d)}$ for various $v^{j}=$ $(c, d)$. For all other $v^{i}=(a, b)$, the surface $S^{\prime}$ will contain no more than $O(N)$ lines representing $\mathcal{T}_{v^{i}, v^{j}}$. Hence, $S^{\prime}$ contains no more than $O(N)$ lies of $L^{\prime}$ altogether.

Condition (ii) can be verified directly. Suppose now $S^{\prime}$ is a fixed plane with the equation $\alpha x_{1}+\beta x_{2}+\gamma x_{3}=\delta$. Thus the quadruple $(\alpha, \beta, \gamma, \delta)$ is fixed up to a multiplier. Fix $v^{i}=(a, b)$. Let $v^{j}=(c, d)$ vary. Let us show that there exists at most one pair $(c, d)=(c, d)\left[a, b, S^{\prime}\right]$, such that the line in $L^{\prime}$, representing $\mathcal{T}_{(a, b),(c, d)}$ lies in $S^{\prime}$. Note that by the initial (generic) rotation assumption none of the $a, b, c, d$ equals zero.

The transformations $T_{i j}$ mapping $v^{i}$ into $v^{j}$ are in the standard basis given by unitary matrices

$$
\left(\begin{array}{ll}
x_{1} & x_{2} \\
x_{3} & x_{4}
\end{array}\right)
$$

such that

$$
\left\{\begin{array}{l}
a x_{1}+b x_{2}-c=0 \\
a x_{3}+b x_{4}-d=0
\end{array}\right.
$$

Suppose $a, b$ are fixed, while $(c, d)$ vary.

Multiply the second equation by $x_{1} \neq 0$ and use $x_{1} x_{4}=1+x_{2} x_{3}$ to eliminate $x_{4}$ from the second equation and then use the first one. We then have a system of three linear equations:

$$
\left\{\begin{array}{l}
a x_{1}+b x_{2} \\
-d x_{1}+c x_{3}=c, \\
\alpha x_{1}+\beta x_{2}+\gamma x_{3}=\delta .
\end{array}\right.
$$

The latter system of equations, with fixed $(a, b)$ has a finite number of solutions which correspond to point intersections of the lines of $L^{\prime}$ with the plane $S^{\prime}$ and are therefore of no interest, unless they are degenerate. Suppose the system of equations (8) is degenerate and has infinitely many solutions. Since $b, c \neq 0$ we cannot have $\gamma, \beta=0$. Let us consider two cases: (i) $\beta=0, \gamma \neq 0$ and (ii) $\beta \neq 0$.

In case (i), we can set $\gamma=1$, which, given $S^{\prime}$, will fix $\alpha, \delta$. If the equations are dependent, we have $(\alpha, 1, \delta) \sim(-d, c,-b)$. This clearly allows for at most one value of $(c, d)=(c, d)\left[a, b, S^{\prime}\right]$.

In case (ii), we can set $\beta=1$, which, given $S^{\prime}$, will fix $\alpha, \gamma, \delta$. If the equations are dependent, we have, for some $\lambda_{1}, \lambda_{2} \neq 0$ :

$$
\lambda_{1} b=1, \quad \lambda_{2} c=\gamma, \quad \lambda_{1} c-\lambda_{2} b=\delta .
$$


This implies that $c$ satisfies $\frac{c}{b}-\frac{b \gamma}{c}=\delta$, which yields no more than two possible values of $c$, and hence (since the set $P$ has been initially rotated to ensure that for each $c$, there is at most one $d$, such that $(c, d) \in P$ ) there are no more than two values of $(c, d)$.

Therefore, in either case $S^{\prime}$ contains no more than $2 N$ lines from $L^{\prime}$.

Thus, the line family $L^{\prime}$ satisfies the assumptions of Theorem 1.1 and this completes the proof of Theorem 1.2.

The proof of Theorem 1.3 repeats the proof of Theorem 1.2, with the only change that one restricts equation (2) to $v^{1}, v^{3} \in P ; v^{2}, v^{4} \in P^{\prime}$. In the trivial case when one does not have $\gg N^{2}$ non-collinear pairs involved, unless both $P$ and $P^{\prime}$ are supported on the same line, there will be a point either in $P$ or $P^{\prime}$, which alone is responsible for $\gg N$ triangles with distinct areas, whose other two vertices are the origin and a point from the counterpart set.

Proof of Corollaries 1 and 1.2. Corollary 1.1 follows from Theorems 1.2 after taking $P=A \times A$ and noticing that the equation (2) then becomes

$$
a_{1} a_{2}-a_{3} a_{4}=a_{5} a_{6}-a_{7} a_{8}, \quad a_{i} \in A, i=1, \ldots, 8,
$$

and the terms can be trivially rearranged to have the plus signs replace the minus signs. The bulk of the proof of Theorem 1.2 furnishes the bound $O\left(|A|^{6} \log |A|\right)$ for the number of solutions of the equation (9), and the claim (1) of the Corollary then follows by Cauchy-Schwartz.

To prove Corollary 1.2 from Theorem 1.3, just set $P^{\prime}=P^{\perp}=\left\{w^{\perp}: w \in P\right\}$.

\section{Acknowledgments}

The authors are indebted T. Tao for a very helpful exposition and discussion of the Guth-Katz theorem and its implications in his blog, and L. Li for having carefully read and commented on the draft. Iosevich was partially supported by the NSF Grant DMS10-45404.

\section{References}

[1] P. Brass, W. Moser and J. Pach, Research problems in discrete geometry, Springer 2005.

[2] D. Covert, D. Hart, D. Koh and M. Rudnev, Generalized incidence theorems, homogeneous forms and sum-product estimates in finite fields, Eur. J. Combinatorics 31 (2010), 306-319.

[3] Z. Dvir, On the size of Kakeya sets in finite fields, J. Amer. Math Soc. 22 (2009), 1093-1097.

[4] G. Elekes and M. Sharir, Incidences in three dimensions and distinct distances in the plane, in 'Proceedings 26th ACM Symposium on Computational Geometry', (2010), 413-422.

[5] G. Elekes, H. Kaplan and M. Sharir, On lines, joints, and incidences in three dimensions, Preprint arXiv:math/0905.1583 (2009).

[6] P. Erdős. On sets of distances of $n$ points, Amer. Math. Mont. 53 (1946), 248-250.

[7] B. Erdogan, D. Hart and A. Iosevich, Multi-parameter projection theorems with applications to sums-products and finite point configurations in the Euclidean setting, Preprint arXiv: math/1106.5544v1 (2011).

[8] L. Guth, The endpoint case of the Bennett-Carbery-Tao multilinear Kakeya conjecture, Acta Math. 205(2) (2010), 263-286.

[9] J. Garibaldi, A. Iosevich and S. Senger, The Erdős distance problem, AMS Student Library Series 56 (2011), 161pp.

[10] L. Guth and N. H. Katz, Algebraic methods in discrete analogues of the Kakeya problem, Adv. Math. 225 (2010), 2828-2839. 
[11] L. Guth and N. H. Katz, On the Erdös distinct distance problem in the plane, Preprint arXiv:math/1011.4105 (2010).

[12] D. Hart, A. Iosevich, D. Koh and M. Rudnev, Averages over hyperplanes, sum-product theory in vector spaces over finite fields and the Erdos-Falconer distance conjecture, Trans. Amer. Math. Soc. 363 (2011), 3255-3275.

[13] R. Pinchasi, The minimum number of distinct areas of triangles determined by a set of $n$ points in the plane, SIAM J. Discrete Math. 22(2) (2008), 828-831.

[14] G. Salmon, A Treatise on the analytic geometry of three dimensions, 2, 5th ed., Hodges, Figgis And Co. Ltd. 1915.

[15] E. Szemerédi and W. T. Trotter, Extremal problems in discrete geometry, Combinatorica 3 (1983), 381-392.

[16] T. Tao and V. Vu, Additive combinatorics, Cambridge University Press, 2006.

Department of Mathematics, University of Rochester, Rochester, Ny 14627, USA

E-mail address: iosevich@math.rochester.edu

Department of Mathematics, University of Bristol, Bristol, BS8 1TW, UK

E-mail address: maorn@bristol.ac.uk, m.rudnev@bristol.ac.uk 
